\title{
HÁBITAT EN EL SALVADOR, PROCESOS Y ESPACIOS QUE LO CONFIGURAN. Propuesta de enfoque de análisis del hábitat, el caso de Arcatao $^{1}$
}

\author{
Habitat in El Salvador, processes and spaces that setting it. Approach proposal \\ for habitat analysis, the Arcatao Case ${ }^{2}$ \\ Grande Ayala, Carlos Ernesto; \\ Universidad Centroamericana José Simeón Cañas, UCA, cgrande@uca.edu.sv
} Cummings, Andrew Robert

Universidad Centroamericana José Simeón Cañas, UCA acummings@uca.edu.sv Manzano Moran, Carlos Alberto; Universidad Centroamericana José Simeón Cañas, UCA, cmanzano@uca.edu.sv Morales Herrera, Herberth Stanley; Universidad Centroamericana José Simeón Cañas, UCA, hmorales@uca.edu.sv

\section{RESUMEN}

La investigación "Procesos sociales y condiciones físico-espaciales que configuran en mayor medida el hábitat en los asentamientos humanos de El Salvador" propone un enfoque de análisis de las condiciones de la producción social del hábitat, a través de un caso de estudio en el municipio de Arcatao. Estructurada a partir del enfoque del pluralismo metodológico crítico, esta investigación, brinda al menos tres resultados claves. Una evaluación con indicadores y cartografía de la producción social del hábitat; Las características principales de los actores, su capacidad de agencia y la forma en que se articulan entre sí; y un análisis histórico de los procesos de producción del hábitat, lo que permite conocer las condiciones estructurales y a capacidad de agencia de un actor o un grupo, necesarias para la emergencia de procesos clave para las transformaciones físico-espaciales del hábitat.

Palabras clave: Producción Social del Hábitat, Asentamientos Humanos, Actores, Capacidad de agencia Bloque temático: Análisis y proyecto territorial

\section{ABSTRACT}

This research is Titled: "Social processes and physical-spatial conditions that configure to a greater extent the habitat in the human settlements of El Salvador. This research proposes an approach to analyze the conditions of the social production of habitat in this country, through a case of study in the municipality of Arcatao at the north east of our country. This research proposal is structured on the approach of "critical methodological pluralism", that allows at least three key results of the municipality studied. First, the evaluation and production of cartography , based on GIS mapping, about the social production of habitat; second, the identification of key stakeholders, their characteristics their agency capability and the sets of networks through which they articulate among themselves; and finally, a historical analysis of the processes regarding the production of habitat, which allows to get a deeper understanding of the key structural conditions and the agency capability of stakeholders, needed for the emergence of the processes underpinning the resulting spatial transformations of the habitat.

Keywords: Social production of the habitat, Human Settlements, stakeholders, Agency capability Topic: Analysis and land project

\footnotetext{
${ }^{1}$ Este proyecto fue financiado con fondos de investigación de la Universidad Centroaméricana José Simeón Cañas, UCA

${ }^{2}$ This Project was funded by research fund of Universidad Centroamericana José Simeón Cañas, UCA.
} 


\section{Introducción}

El hábitat, entendido tanto desde una perspectiva de desarrollo sostenible como de derecho, se ha posicionado como un tema de discusión relevante en el ámbito político internacional. Esto se debe principalmente al rol estratégico de las ciudades del s. XXI y a la acelerada tendencia con la cual éstas aumentan de número y dimensión. Esta situación trae consigo nuevas tensiones sociales por el espacio. Es frente a esta problemática que existen directrices internacionales comprometidas con la generación de un hábitat digno que promuevan el mejoramiento de la calidad de vida desde un enfoque de sostenibilidad (Hábitat; Naciones Unidas, 2016), así como objetivos e indicadores que plantean metas claras y específicas en materia de mejoramiento de hábitat y disminución de la pobreza.

El Salvador también ha experimentado procesos donde el concepto de hábitat ha sido replanteado a partir de un ejercicio participativo, verbigracia, la formulación de la Política Nacional de Hábitat y Vivienda y la propuesta de Ley de Hábitat y Vivienda (PNHV) (MOPTVDU, 2015), la cual permite el desarrollo de iniciativas que den respuesta a la "compleja problemática que representa la vivienda y el hábitat" en nuestro país, es así que esta investigación busca aportar a esta problemática planteándose el objetivo de comprender y explicar cómo los procesos sociales posibilitan la transformación de las condiciones físico-espaciales y los procesos de producción de las infraestructuras que mejoran el hábitat en los asentamientos humanos de El Salvador, aplicando un esquema de análisis diseñado para El Salvador y aplicado a un caso particular en el norte del país, en concreto el municipio de Arcatao en Chalatenango.

\section{Marco teórico}

En El Salvador el concepto de hábitat se encuentra plasmado en la PNHV, la cual parte de la concepción del hábitat como «Conjunto de infraestructuras de servicios, gestión del riesgo y espacios públicos que aseguran la calidad de vida de los habitantes de acuerdo con su localización en el territorio. Ello incluye los servicios tales como la provisión, disposición y tratamiento del agua, suministro de energía, telecomunicaciones y transporte; equipamientos sociales de salud, educación, cultura, recreación y para actividades económicas» (MOPTVDU, 2015. p.39). A partir de este concepto se establecen grupos de infraestructuras del hábitat que se muestran en el Figura 1, al que se ha incorporado el grupo denominado "Saneamiento ambiental", ya que no hay referencia explícita a algunas de ellas y como refleja Diego Carrillo (2007, p. 53) estas infraestructuras son fundamentales en el hábitat junto con la vivienda. Sin embargo, este concepto solo aporta definiciones descriptivas de los elementos físico-espaciales, para esta investigación, también es relevante comprender ¿Cuáles son los factores determinantes que explican la concreción de ese "conjunto de infraestructuras" y que causa que esos procesos sociales se vuelvan posibles?

Por lo que se realiza un salto cualitativo en la búsqueda de una definición más descriptiva, no solo del hábitat, sino de los procesos sociales que la generan para "explorar en conceptualizaciones que se esfuercen en pronunciarse sobre los mecanismos que producen el curso de los eventos" (Danermark, Ekström, Jakobsen, \& Karlsson, 2016).

\subsection{Del hábitat hacia los procesos de producción del hábitat}

Con esta nueva búsqueda, se traza una ruta de diversas acepciones que en la historia han hecho hincapié en el término "hábitat" desde una perspectiva más compleja y si se quiere explicativa de la existencia del fenómeno. Desde Latinoamérica el término hábitat surge como contraposición a la construcción formal de la ciudad y por mucho tiempo se entendió como las condiciones de habitar propias de las personas en los asentamientos humanos autoconstruidos. Para esta tarea de reconstrucción histórica se tiene como referencia el texto de Miranda Gassull (2017), sintetizado en la Tabla 1, el cual postula cuatro enfoques desde donde se ha abordado el tema del hábitat y que para efectos de esta conceptualización se considera útil porque en conjunto brindan insumos sobre el componente de procesos sociales de producción del hábitat. 


\section{Infrestructuras del hábitat}

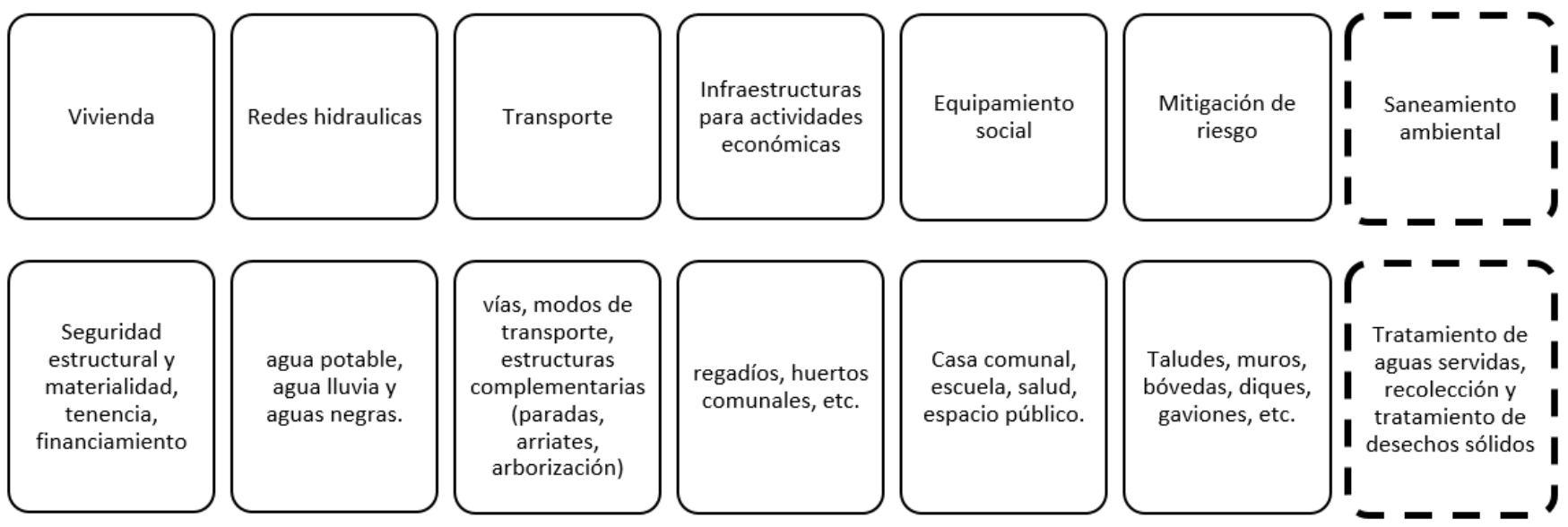

Figura 1: El hábitat y las infraestructuras que lo definen; Fuente: elaboración propia con base en (MOPTVDU, 2015)

\begin{tabular}{|c|c|c|c|c|c|}
\hline $\begin{array}{l}\text { Enfoque } \\
\text { latinoamericano }\end{array}$ & $\begin{array}{l}\text { Habitar promotor } \\
\text { del desarrollo }\end{array}$ & $\begin{array}{l}\text { Habitar } \\
\text { técnica }\end{array}$ & solución & $\begin{array}{l}\text { Habitar: Vivir bien y } \\
\text { el buen vivir }\end{array}$ & Derecho a habitar \\
\hline $\begin{array}{l}\text { Acciones que detonan } \\
\text { procesos sociales }\end{array}$ & $\begin{array}{l}\text { Consumo } \\
\text { energético, } \\
\text { Urbanización y sus } \\
\text { características }\end{array}$ & $\begin{array}{l}\text { Proceso de } \\
\text { fundamentalmente } \\
\text { cuantitativo } \\
\text { Proceso de diseñ } \\
\text { enfoque técnico-social }\end{array}$ & $\begin{array}{l}\text { diseño } \\
\text { técnico- } \\
\text { ño con } \\
\text { l }\end{array}$ & $\begin{array}{l}\text { Organización } \\
\text { comunitaria-ecológica. }\end{array}$ & $\begin{array}{l}\text { Organización social } \\
\text { fundamentada en } \\
\text { reivindicación de los } \\
\text { derechos humanos. } \\
\text { Procesos de } \\
\text { investigación }\end{array}$ \\
\hline
\end{tabular}

Tabla 1: Acciones que determina procesos sociales que dan forma al hábitat. Fuente: Elaboración propia con base en (Miranda Gassull, 2017)

Un primer enfoque denominado «habitar promotor del desarrollo» tiene su origen con la conferencia promovida por la ONU sobre Asentamientos Humanos 1976, ahí "se comienza a instalar el término hábitat como enfoque multidimensional superador al de vivienda». Un segundo enfoque proviene de la arquitectura, «habitar como solución técnica» influenciado por la vivienda de Le Corbusier entendida como "máquina de habitar" que imprimió un fuerte componente físico-espacial a la idea de calidad de la vivienda, sin embargo, Miranda plantea la crisis de este paradigma por el fracaso del modelo del estado benefactor como proveedor de vivienda y desde entonces, principalmente desde la academia, se asume un rol más crítico hacia las políticas bajo esta perspectiva.

Un tercer enfoque es "el habitar desde la concepción andino-indígena", aquí la vida es concebida como un todo indivisible y los conceptos de comunidad y relación armónica con la naturaleza guían la obtención de nuevas formas de espacios habitables. Finalmente, el cuarto enfoque, "El derecho a habitar", plantea la relación del hábitat con la "experiencia sociopolítica que implica habitar", coloca como principales impulsores a "diferentes movimientos y organizaciones sociales de base, activistas de derechos humanos y grupos académicos" (Rodríguez et al., 2007) y en las cuales se inicia la idea de "hábitat de producción social", que propone la existencia de diversas fuerzas sociales interactuantes con el modo de habitar, destacándose los actores que reproducen lógicas propias.

Para El Salvador este última idea es adaptada por Carrillo (2007), quien adherido a la idea de (Ortíz \& Zárate, 2002) plantea que la producción social del hábitat se debe a «Todos aquellos procesos generadores de espacios habitables, componentes urbanos y viviendas que se realizan bajo el control de auto productores y otros agentes sociales que operan sin fines lucrativos». Ambos hablan de "procesos" aunque no explícitamente; los términos "operaciones necesarias" en Carrillo y "autoproducción y agentes sociales" de Ortiz perfilan el importante aporte del componente social a la definición de los espacios habitables, que Lefebvre (2013, p. 14) había anticipado considerando que "El espacio es el resultado de la acción social, de las prácticas, las relaciones, las 
experiencias sociales, pero a su vez es parte de ellas", es esta relación de causalidad multidimensional del espacio con la acción social, práctica, relaciones y experiencias que permite definir la idea de una categoría de análisis que en esta investigación se denomina "procesos sociales".

\subsection{Los estratos del hábitat}

Los procesos sociales y las determinantes físico-espaciales que configuran el hábitat discurren en tres grandes dimensiones (espacio concebido, percibido y vivido) que dan forma a «una teoría unitaria del espacio, dada esa diversidad y fragmentación, y en concreto, tras la constatación de una contradicción diabólica entre la percepción, concepción y vivencia" (Lefebvre, 2013, p. 47).

Lo relevante de esa teoría es la asociación que hace de cada dimensión del espacio con "el espacio" como unidad de análisis (véase Figura 2) lo cual dota a la propuesta teórica de unas "características del espacio muy concretas" que aunque nunca se experimentan de manera individual o separadas en la realidad, permite construir una estructura de relaciones del fenómeno lo suficientemente clara para concretizar un análisis estratificado de la realidad espacial (Danermark et al., 2016).

\subsection{Aportes teóricos para el hábitat en El Salvador}

Para El Salvador la corriente del hábitat denomina "El derecho a habitar" ha sido estudiada, estructurada y adaptada por Carrillo (2007), y tiene implícitos procesos de autogestión y por lo tanto apuesta por un hábitat impulsado por acciones concretas desde la sociedad. Carrillo no hace referencia explícita a la teoría unitaria del espacio, sin embargo, se considera que las tres dimensiones o momentos del espacio propuestas por Lefebvre tienen muchas coincidencias mutatis mutandi con respecto a la propuesta de ámbitos del hábitat que realiza Carrillo para El Salvador, esto es relevante por cuatro aspectos, primero aporta nuevos procesos sociales que intervienen en la construcción del hábitat, segundo facilita la operativización de las variables de estudio (véase Tabla 2) ya que cada área de acción posee diversas propuestas metodológicas para ser evaluadas y por lo tanto analizadas; tercero, Integra a la valoración del hábitat aspectos cualitativamente distintos como infraestructuras y procesos sociales, finalmente hay una construcción conceptual desde la realidad de El Salvador lo que permite hacer bastantes precisiones del contexto del hábitat en nuestro país.

\begin{tabular}{|c|c|c|c|}
\hline \multirow{15}{*}{. } & & Acción & Indicador específico \\
\hline & \multirow{4}{*}{ Socio - Cultural } & Educación & Proximidad de escuela \\
\hline & & Salud & Proximidad de clínica \\
\hline & & Medio Ambiente & Tratamiento de \\
\hline & & ${ }^{*}$ Seguridad & Presencia de pandillas o maras \\
\hline & \multirow[t]{7}{*}{ Físico Material } & Vivienda & Vivienda \\
\hline & & \multirow{6}{*}{$\begin{array}{l}\text { Infraestructura físico y } \\
\text { social }\end{array}$} & Vías \\
\hline & & & Drenajes \\
\hline & & & Sistema de redes de abasto (Energia Eléctrica, Agua) \\
\hline & & & Recolección de desechos sólidos \\
\hline & & & Transporte \\
\hline & & & Equipamiento Social (casa comunal, espacio público) \\
\hline & \multirow[t]{3}{*}{ Económico } & Empleo & Organización e infraestructura \\
\hline & & Legalización & Tenencia de la tierra \\
\hline & & Financiamiento & ONG, Estado, Municipio, etc. \\
\hline
\end{tabular}

Tabla 2: variables de estudio preliminares de procesos e infraestructuras del hábitat, fuente: Elaboración propia con base en (Ca millo, 2007)

Dicho lo anterior se enuncian los "tres ámbitos del hábitat en los que es posible organizar nueve áreas de acción que giran en torno al problema del hábitat, según el constructo teórico de Carrillo (2007, p. 30), primero el ámbito Físico -Material que abarca: vivienda e Infraestructura física y social, segundo el ámbito Económico que implica la legalización, financiamiento y empleo y finalmente el ámbito Socio Cultural que incluye Educación, medioambiente y salud los cuales a su vez se equiparan con las dimensiones del espacio de Lefebvre (véase Figura 2) de tal manera que se constituye un esquema preliminar de relaciones y procesos internos al fenómeno de construcción del hábitat, esta equiparación no implica en modo alguno la contención de las Acciones del hábitat en una Categoría de Espacio, sino que persigue una análisis más holístico de determinada Acción del hábitat en el estudio del fenómeno de la producción social del espacio. 


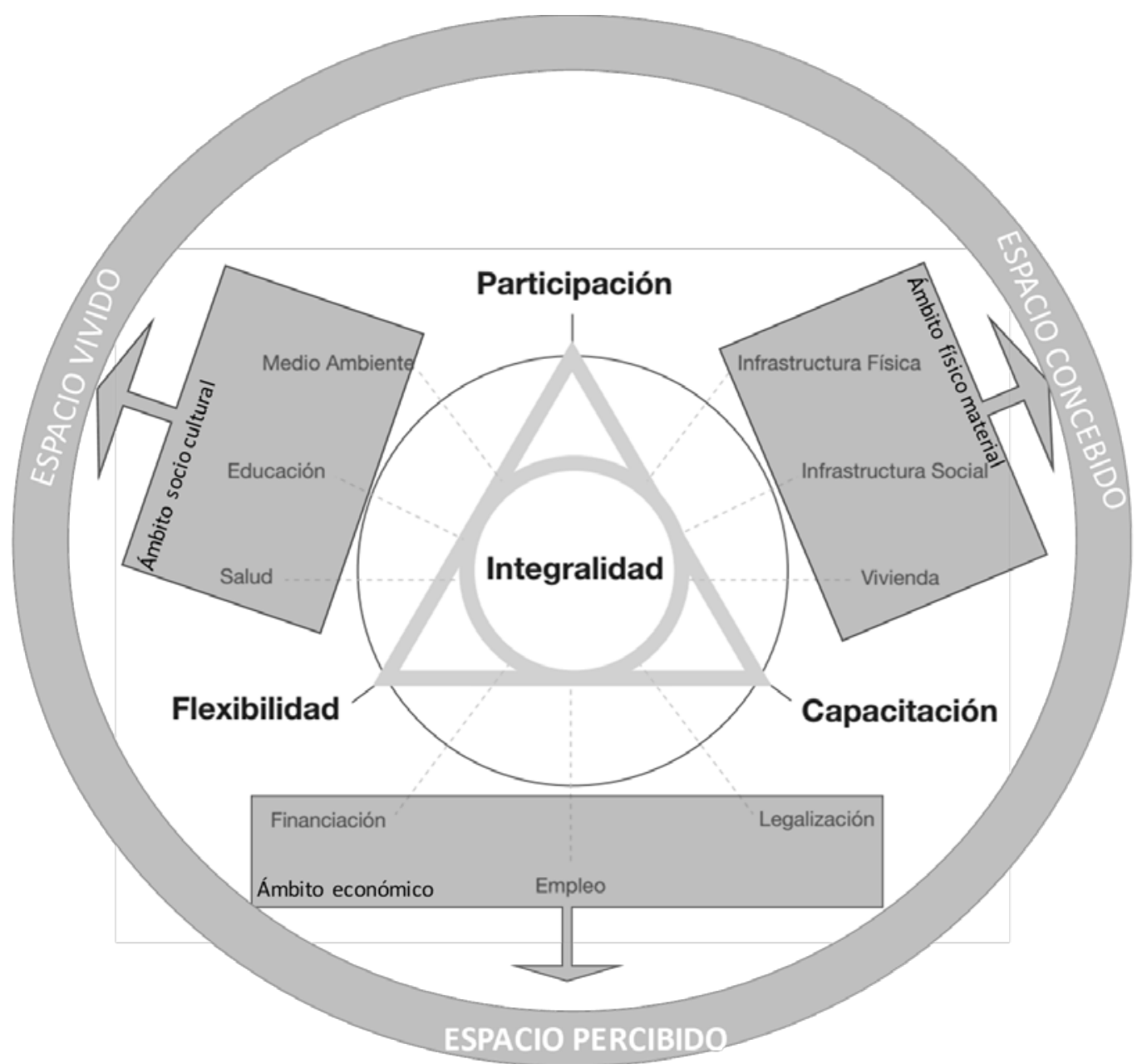

Figura 2: Dimensiones del Espacio de Lefebvre 2013 y Ámbitos del hábitat de Carrillo, 2007 ; Fuente: Elaboración propia

\subsection{El "lugar del hábitat"}

Para entender el hábitat, es necesario establecer una conceptualización orientadora para la delimitación del espacio sobre el cual se constaten las hipótesis planteadas, es así como, la idea de asentamientos humanos permite delimitar la unidad de análisis del objeto de estudio. Para El Salvador esto implica una limitante importante ya que la última unidad geográficamente delimitada a nivel de censo es el cantón, pero es posible delimitar unidades más pequeñas y de forma más estratégica se identifica la escala de asentamiento humano (que podría tener diferentes nombres i.e. colonia, lotificación, comunidad, etc.), pues es en esta unidad en la que se dan los emprendimientos más importantes de los procesos sociales del hábitat.

Considerando lo anterior y que en El Salvador no existe una definición satisfactoria de Asentamientos Humanos se parte de las ideas expuestas en la Política de Desarrollo Territorial (MOPTVDU, 2007) y la PNHV y los criterios provenientes del Desarrollo Rural (Soto Baquero, Beduschi Filho, Diez de Sollano, Valenzuela Chadwick, \& Palma, 2007) para proponer una definición operativa en la cual se establezca como Asentamientos Humanos a las "áreas poblacionales ubicadas espacial, física, cultural y socialmente en un territorio delimitable a partir de criterios geográficos, étnico-culturales y factores económico-sociales". Esto constituye un concepto orientador para poder delimitar y definir geográficamente los asentamientos humanos como unidades de análisis del hábitat. 


\subsection{Condicionantes físico-espaciales, procesos sociales y la representación del fenómeno del hábitat}

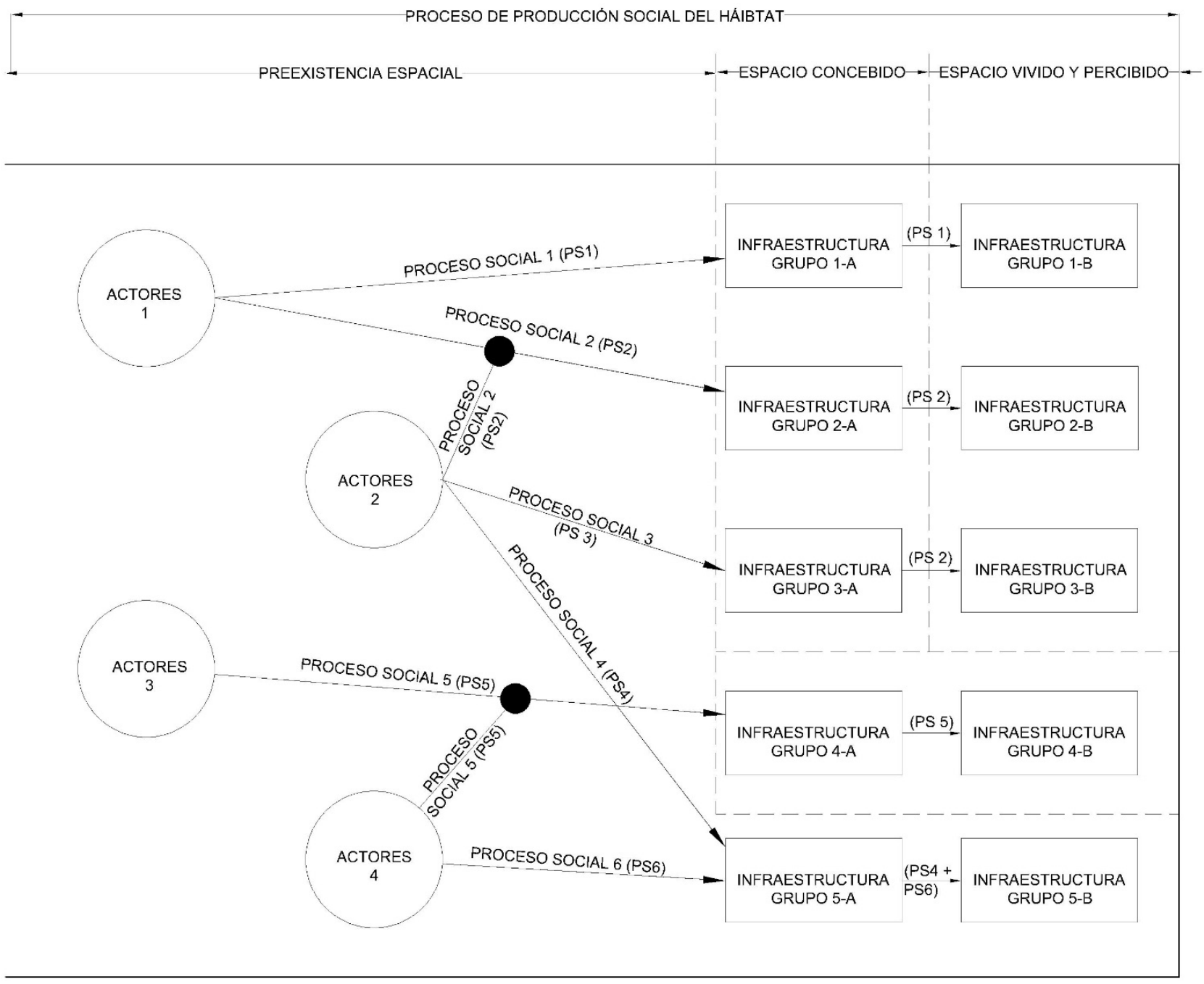

Figura 3: Proceso de producción social del hábitat; Fuente: Elaboración propia.

En este contexto la Figura 3 plantea la hipótesis sobre la que se basa esta investigación, tiene como base dos conceptos propuestos por Lefebvre, el primero sobre la teoría unitaria del espacio (espacio concebido, vivido y percibido) (2013, p. 72) y la idea de preexistencia espacial (2013, p. 115), sentando así la premisa hipotética para definir lo que se denomina condicionantes físico-espaciales, que a pesar de las fuerzas producidas por los procesos sociales en algunos casos son difíciles de transformar y en ocasiones, la producción social logra adaptar al hábitat a esta condicionante, además se incorpora la idea de actores que según sus características e intereses desarrollan procesos sociales (véase Tabla 3) que tienden a transformar la preexistencia espacial para volverlo lugar de condiciones apropiadas, contrastando así con la visión tecnocrática predominante en intervenciones de mejora del territorio. Finalmente estos procesos sociales llevan a un estado de transformación de la preexistencia espacial a través de la implantación de infraestructura, moviéndose para ello desde la preexistencia, pasando por el espacio concebido (o lo que sería un estadio de planificación y diseño) hasta la concretización de esos proyectos habilitando el espacio vivido y percibido, estas últimas dos categorías además no excluyen la posibilidad de encontrar en formas muy puras infraestructuras poco o nada transformadas del espacio concebido. 


\begin{tabular}{|l|l|}
\hline \multicolumn{1}{|c|}{ Proceso social vinculado al hábitat } & \multicolumn{1}{|c|}{ Fuente } \\
\hline Consumo energético & (Miranda Gassull, 2017, p. 222) \\
\hline Proceso de urbanización & $\begin{array}{l}\text { (Carrillo, 2007, Capitulo 3; Miranda Gassull, 2017, p. } \\
\text { 222) }\end{array}$ \\
\hline $\begin{array}{l}\text { Proceso de diseño (con enfoque técnico cuantitativo o técnico-social) } \\
\text { Organización comunitaria (con enfoque medioambiental o de reivindicación de } \\
\text { derecho) }\end{array}$ & (Miranda Gassull, 2017, p. 225) \\
\hline Educación & (Carrillo, 2007, p. 33) \\
\hline Salud & (Carrillo, 2007, p. 34) \\
\hline Construcción de vivienda (seguridad estructural, material, legalidad) & (Carrillo, 2007, Capitulo 4) \\
\hline Construcción de infraestructuras del hábitat que no sean vivienda. & (Miranda Gassull, 2017, p. 224) \\
\hline Financiamiento & (Carrillo, 2007, p. 31) \\
\hline Empleo & (Carrillo, 2007, p. 32) \\
\hline Autogestión & (Carrillo, 2007, p. 23) \\
\hline
\end{tabular}

Tabla 3: Síntesis de procesos sociales en el hábitat; fuente: elaboración propia con base en (Carrillo, 2007; Miranda Gassull, 2017)

\section{Metodología}

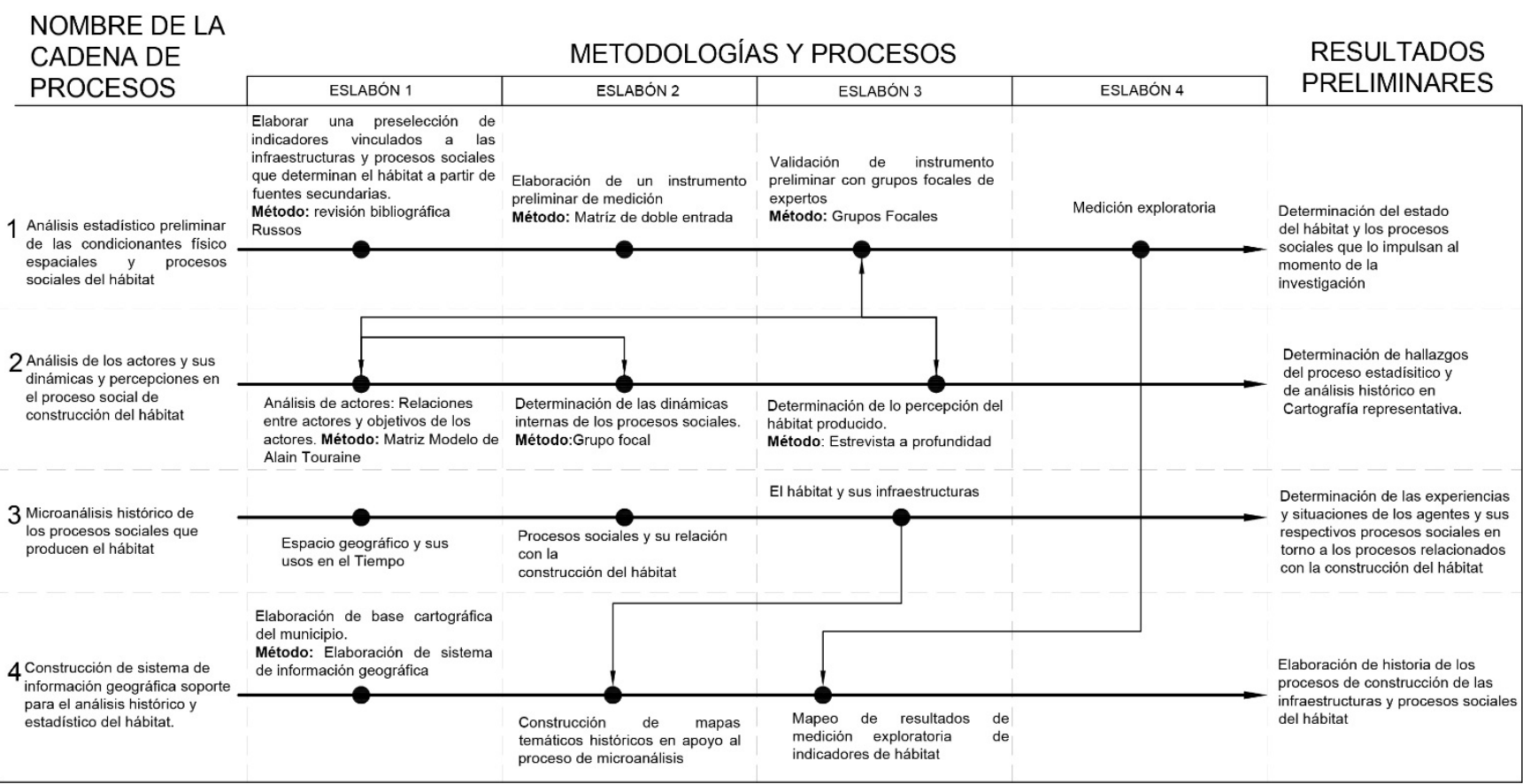

Figura 4: Diseño metodológico de la investigación, Fuente Elaboración propia

El diseño metodológico utilizado dividió la investigación en cuatro ejes interconectados, esto se muestra en la figura 4, el primero, de carácter cuantitativo construyó un sistema de indicadores que evalúan el hábitat desde la visión de la producción social del espacio de Lefebvre y las acciones que fortalecen el hábitat propuestas por Carrillo (2007), dicho proceso inició con una revisión bibliográfica sistematizada (Roussos, 2011), posteriormente se transforma en indicadores(Cárdenas et al., 2013) que son estructurados con ayuda del marco lógico(Ortegón, Pacheco, \& Prieto, 2005), permitiendo generar una batería ampliada de indicadores depurada con la técnica de triangulación (Zúñiga, 2009) lo que dio como resultado un Instrumento Preliminar de Evaluación del Hábitat (IPEHa); con dicho instrumento se realizó un taller de validación con grupos de expertos en hábitat en el país utilizando la metodología Delphi (Peñalva, de los Rios, Aguilera, \& Eraso, 2014), esto permitió tener una versión final del Instrumento de Evaluación de la Producción del Hábitat, el cual fue utilizado para hacer la evaluación del hábitat en el Municipio de Arcatao en el norte del país, utilizando para ello recopilación de datos primarios como la alcaldía municipal, la policía nacional civil, las clínicas de salud y los juzgados, y datos secundarios del censo nacional. 
Un segundo eje de investigación elabora un mapa de actores, define sus características y capacidades de agencia en los procesos sociales que producen el hábitat, utilizando entrevistas a profundidad (Holstein \& Gubrium, 1995) y grupos focales, estos insumos permitieron al menos dos productos, el primero fue sistematizado mediante el análisis temático (Braun \& Clarke, 2006) con el fin de determinar una lectura sobre la capacidad de agencia de los actores utilizando como base los planteamientos conceptuales propuestos por Amartya Sen (1982), dando como resultado matrices y diagramas que buscan mapear los actores presentes en el territorio considerando también su capacidad de agencia. Mientras que el segundo producto consistió en el análisis de redes de actores y la forma en que se vinculan en proyectos de desarrollo del hábitat contrastándola finalmente con la percepción y vivencia del espacio por parte de los pobladores.

El tercer eje es sobre historia, se utiliza la metodología del microanálisis (Aguirre, 2003) y se establece un período que va desde 1980 hasta la actualidad (conflicto armado, pueblo fantasma, repoblación y reconstrucción), permite comprender la influencia de la estructura social en la transformación del hábitat y las capacidades reales de agencia de los actores siguiendo la idea de Archer (1995), las técnicas utilizadas fueron la revisión documental y la observación participante (O'toole \& Were, 2008) obteniendo como resultado final un relato que articula la historia reciente de Arcatao y tiene como eje la producción social del espacio y las categorías planteadas por Lefebvre y Carrillo.

Finalmente un cuarto eje de sistemas de información geográfica inicia con visitas de campo utilizando imágenes de drone, gps y talleres de validación con responsables de catastro de la municipalidad para validar los asentamientos humanos y los límites del término municipal, esta base cartográfica permitió al menos tres productos, el primero, la interpolación del resultados de la evaluación del hábitat a través método IDW (Inverse Distance Weighting) aplicado en variables sociales y de territorio (Espinosa \& Valderrama, 2011), esto permite visualizar la dinámica de la producción social del hábitat en el territorio poniendo especial énfasis en la distribución de la desigualdad del hábitat en los asentamientos humanos. Un segundo producto, genera estudios de evaluación de accesibilidad a través de la herramienta Network Analysis de GIS que son insumos para la evaluación cuantitativa y finalmente se elaboraron mapas de apoyo a la narrativa histórica todos los cuales constituyen el Atlas de cartografía de la investigación.

\section{Resultados}

La primera parte aportó un instrumento con 19 indicadores asociados a 175 subindicadores de evaluación de la producción social del hábitat (véase Tabla 4) con los que se realizó la evaluación del municipio de Arcatao con dicho instrumento, esa evaluación fue utilizada para la generación de mapas, mostrados en la figura 5, estos exponen cómo se distribuye el índice de producción social del hábitat y sus respectivos ámbitos en el territorio de Arcatao. A partir de esta evaluación de 6.91 para el municipio de Arcatao es posible ubicarlo en el rango superior intermedio y se puede concluir que los procesos e infraestructuras son estables y actualmente están funcionando con tendencia a mejorar la calidad de vida en los asentamientos humanos, pero con una marcada desigualdad hacia el sur producto de la complicada topografía de los asentamientos en esa zona, las deficiencias en la red vial y de aguas negras. Este valor es además muy significativo, al contrastarlo con el índice de desarrollo humano (IDH) que coloca a este municipio con un valor de 0.628 que es muy cerca del valor promedio de los municipios del país, esto es 0.68 .

En cuanto a los resultados de la evaluación los actores, sus redes y capacidad de agencia, posterior al mapeo general de los actores presentes en el territorio y a la sistematización de entrevistas se obtuvieron esquemas síntesis de la capacidad de agencia de los actores más relevantes, aquí se muestra la figura 6 de los tres actores considerados claves y sus destacadas valoraciones en el tema de conocimiento de la realidad, liderazgo y recursos, como elementos significativos para valorar su capacidad de agencia. 


\begin{tabular}{|c|c|c|c|c|c|c|c|c|c|c|c|c|c|c|c|c|c|c|c|c|c|c|}
\hline \multicolumn{9}{|c|}{ Índice del Ámbito Físico Material } & \multirow[b]{3}{*}{ 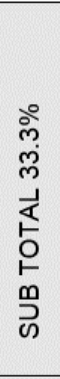 } & \multicolumn{7}{|c|}{ Índice del Ámbito Económico } & \multicolumn{5}{|c|}{$\begin{array}{c}\text { Índice del Ámbito Socio } \\
\text { Cultural }\end{array}$} & \multirow{3}{*}{ 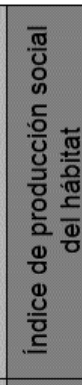 } \\
\hline \multirow[b]{2}{*}{ 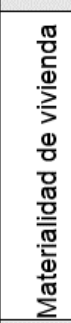 } & \multirow[b]{2}{*}{ 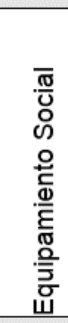 } & \multicolumn{5}{|c|}{ REDES } & \multirow[b]{2}{*}{ 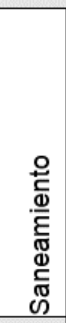 } & \multirow[b]{2}{*}{ 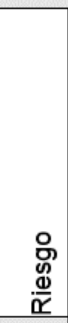 } & & \multirow{2}{*}{ 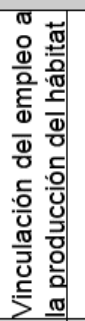 } & \multirow[b]{2}{*}{ 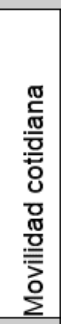 } & \multirow[b]{2}{*}{ 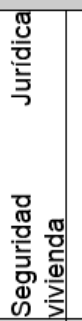 } & \multirow[b]{2}{*}{ 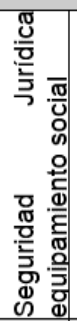 } & \multirow[b]{2}{*}{ 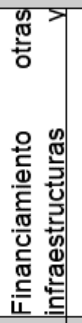 } & \multirow{2}{*}{ 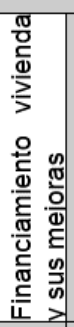 } & \multirow[b]{2}{*}{ 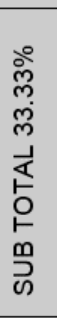 } & \multirow{2}{*}{\multicolumn{2}{|c|}{ 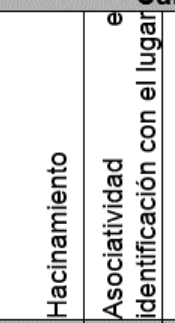 }} & \multirow[b]{2}{*}{ 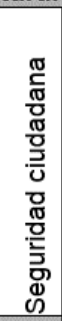 } & \multirow[b]{2}{*}{ 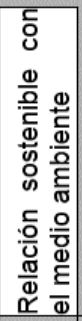 } & \multirow[b]{2}{*}{ 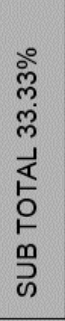 } & \\
\hline & & $\begin{array}{l}\bar{\sigma} \\
\overline{>} \\
\overline{0} \\
\mathbb{\Phi} \\
\underline{\underline{y}}\end{array}$ & 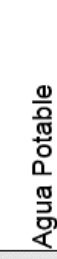 & 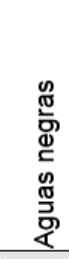 & 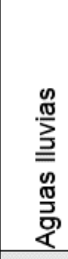 & 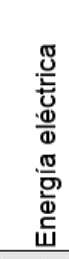 & & & & & & & & & & & & & & & & \\
\hline $1.1 \%$ & $1.1 \%$ & $1.1 \%$ & $1.1 \%$ & $1.1 \%$ & $1.1 \%$ & $1.1 \%$ & $1.1 \%$ & $1.1 \%$ & $00 \%$ & $6.7^{\circ}$ & $16.7 \%$ & $16.7 \%$ & $16.7 \%$ & $16.7 \%$ & $16.7 \%$ & 100 & $5.0 \%$ & $25.0 \%$ & $25.0 \%$ & 25.0 & 100 & \\
\hline
\end{tabular}

Tabla 4: Indicadores que forman para evaluación del hábitat, Fuente: elaboración propia

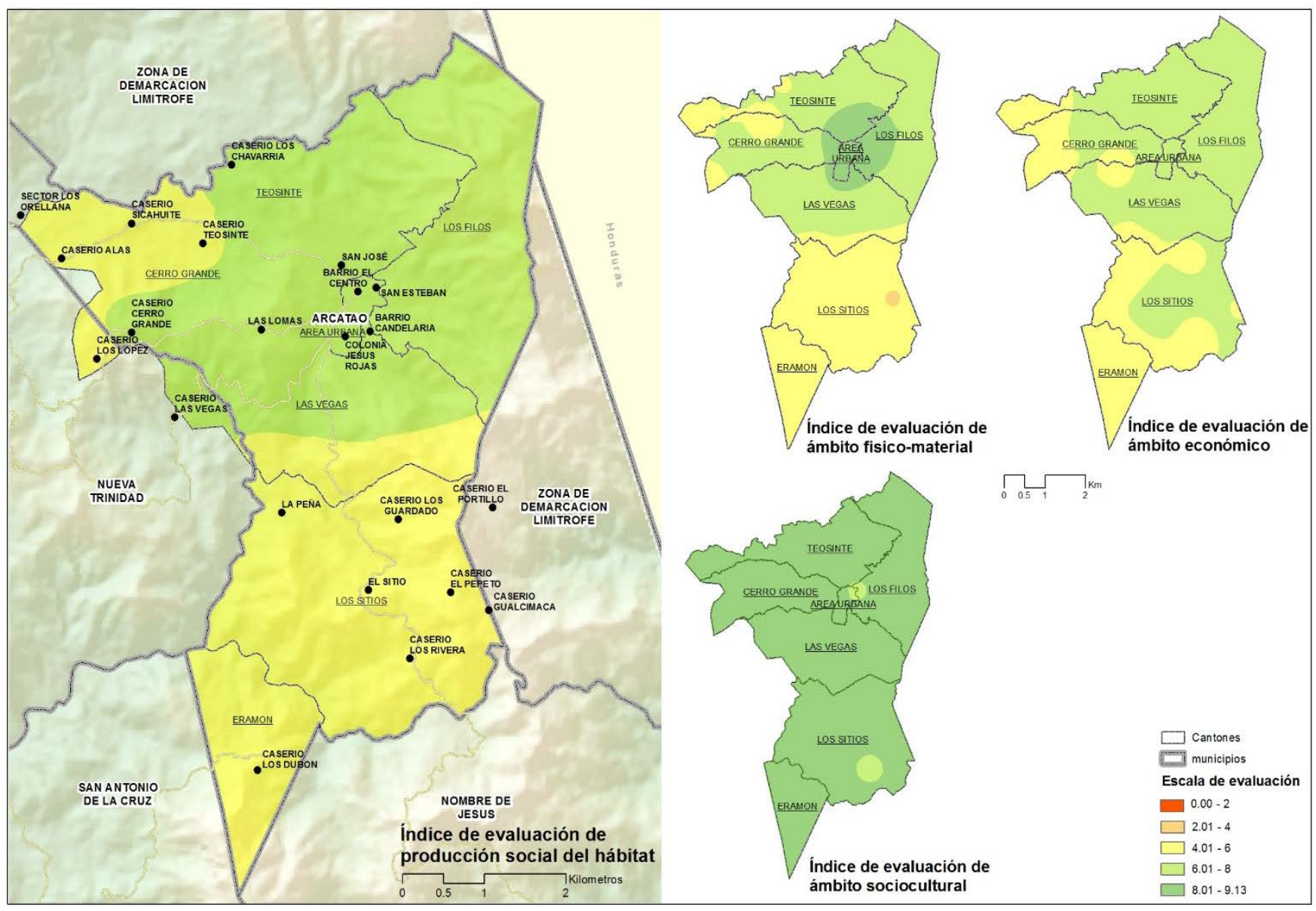

Figura 5: Interpolación de valores del hábitat en Arcatao; Fuente: Elaboración propia 


\begin{tabular}{|c|c|c|c|c|c|c|c|c|c|c|c|}
\hline \multicolumn{4}{|c|}{ Coordinadora de Comunidades Rurales (CCR) } & \multicolumn{4}{|c|}{ Parroquia San Bartolomé } & \multicolumn{4}{|c|}{ Alcaldia Municipal de Arcatao } \\
\hline \multicolumn{2}{|c|}{ Posición } & \multicolumn{2}{|c|}{ Rol } & \multicolumn{2}{|c|}{ Posición } & \multicolumn{2}{|r|}{ Rol } & \multicolumn{2}{|c|}{ Posición } & \multicolumn{2}{|c|}{ Rol } \\
\hline Presencio & $\begin{array}{l}\text { Nivel de } \\
\text { actuación }\end{array}$ & Sector & Ámbito & Presencia & $\begin{array}{l}\text { Nivel de } \\
\text { actuación }\end{array}$ & Sector & Ámbito & Presencia & $\begin{array}{c}\text { Nivel de } \\
\text { actuación }\end{array}$ & Sector & Ámbito \\
\hline Interna & Local & Social & $\begin{array}{l}\text { Organización, } \\
\text { educación }\end{array}$ & Interna & $\begin{array}{l}\text { Local, } \\
\text { Regional }\end{array}$ & Social & $\begin{array}{c}\text { Culto, } \\
\text { Organización, } \\
\text { Act. Productivas }\end{array}$ & Interna & Local & $\begin{array}{c}\text { Gobierno } \\
\text { local }\end{array}$ & $\begin{array}{l}\text { Gestión, } \\
\text { organización, } \\
\text { construcción, } \\
\text { financiamiento }\end{array}$ \\
\hline
\end{tabular}

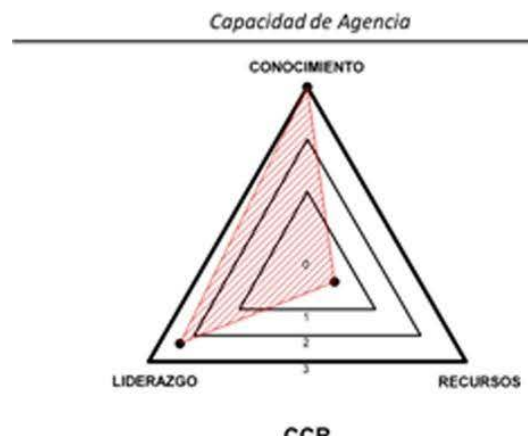

Capacidad de Agencia

Capacidad de Agencia

CCR

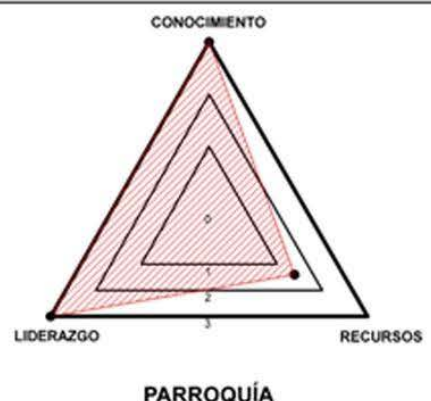

PARROQUIA

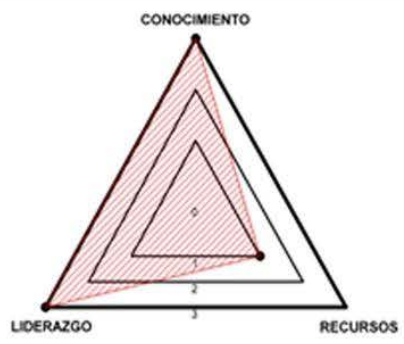

ALCALDIA

Figura 6: Análisis de capacidad de agencia de los actores en Arcatao, Fuente Evaluación propia

Adicionales a los gráficos por actor, se construyó una matriz que busca ser el inicio de un mapa de actores que permita, no solo posicionar a cada actor en su ámbito de acción y sector respectivo, sino completar dicha lectura con la capacidad de agencia del mismo lo que permite una lectura más completa sobre la composición, distribución y fortalezas de los actores presentes en el territorio analizado (véase Tabla 5). Asimismo, bajo la premisas conceptuales que los actores identificados son altamente dinámicos y que el capital social presente en el territorio es clave para la transformación del mismo, se desarrollaron análisis de redes a partir del enfoque analítico presentado por Scott (2017). En la figura 7 por ejemplo, es posible observar la centralidad de los actores clave (CCR, Alcaldía y Parroquia) y sus respectivos "clusters" o sistemas cíclicos relacionales donde se evidencian nodos donde en la praxis confluye información y recursos

\begin{tabular}{|c|c|c|c|c|c|c|c|c|}
\hline & \multicolumn{7}{|c|}{ Ámbito } \\
\hline & & Educación & Salud & Construcción & Organización & Act. Productivas & Medio Ambiente & Otro \\
\hline \multirow{11}{*}{ 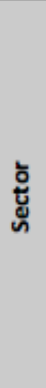 } & \multirow{2}{*}{ Publico } & & & & & & & \\
\hline & & & & Alcaldía & Alcaldía & & & Alcaldía \\
\hline & \multirow{2}{*}{ Privado } & & & & & & & \\
\hline & & UCA-Chalatenango & & & & & & \\
\hline & \multirow{7}{*}{ Social } & & & & & & & \\
\hline & & Plan Internacional & & & & & & \\
\hline & & ACISAM & & & & & & \\
\hline & & CBC & & & $\mathrm{CBC}$ & & & \\
\hline & & Parroquía* & & & Fundación Mártires & $\mathrm{CBC}$ & & CORDES \\
\hline & & CORDES & CBC & Plan Internacional** & Parroquía & Parroquía & Plan Internacional & CBC \\
\hline & & CCR & ACISAM & CORDES** & $\mathrm{CCR}$ & CORDES & CORDES & ACISAM \\
\hline
\end{tabular}

*Culto, formación política

** Mejoramiento físico
Alta capacidad de agencia Moderada capacidad de agencia Baja capacidad de agencia

Tabla 5: Relaciones entre actores y capacidad de agencia; Fuente Elaboración propia 


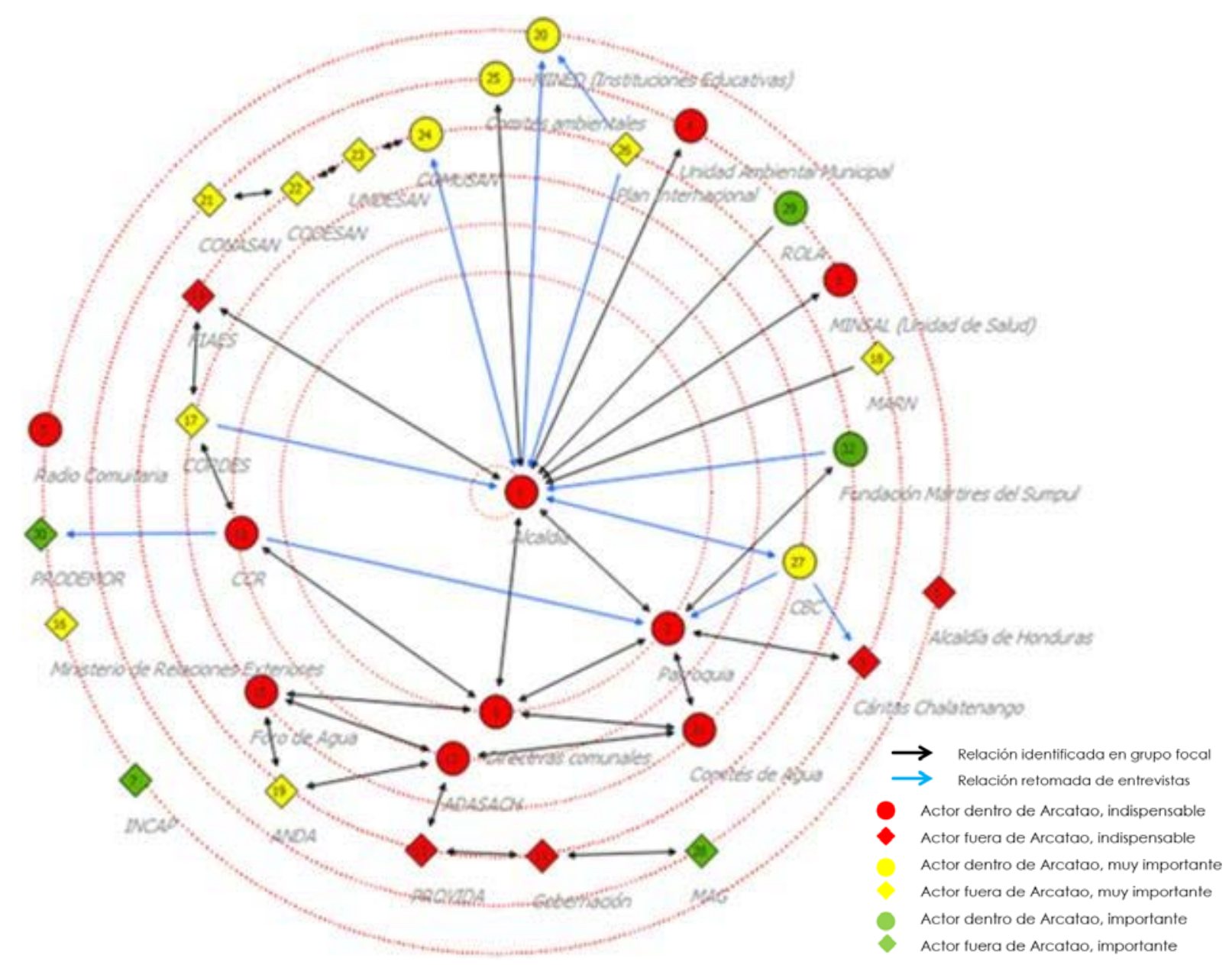

Figura 7: Relación de actores en torno al tema de medio ambiente y agua; Fuente: Elaboración propia

En cuanto al análisis histórico, los resultados consisten en una narrativa que articula los sucesos relevantes de la producción social del hábitat la cual consta de los siguientes apartados; primero el espacio como producto social, establece una cronología de hechos fundacionales e hitos históricos del municipio previo al inicio del período de estudio citado con anterioridad. Segundo y tercer aspecto es lo relacionado con la espacialidad del conflicto armado y el posterior despoblamiento y desplazamiento de la población, que describe cómo la guerra da forma a un Arcatao más itinerante y en constante huida producto de las incursiones militares que amenazaban con la vida de los pobladores, un cuarto momento describe eventos y procesos que favorecieron el paulatino repoblamiento posterior al conflicto y ubica la creación de importantes programas con suficiente fundamento para ir mejorando necesidades básicas como salud y educación aún sin contar con ayuda de la alcaldía o gobierno central, finalmente se aborda la reconstrucción con el relato de los procesos más relevantes en el contexto de la post guerra a nivel nacional, destacando la forma en que la organización comunal de Arcatao enfrenta ese proceso de manera crítica y crear mejores condiciones para inversión y captación de fondos incluso de la ayuda internacional en la producción de su hábitat.

Finalmente se trabaja de forma paralela apoyando en la construcción de cartografía y realizando análisis espacial como el que se muestra en la Figura 5 y de apoyo al análisis histórico como la definición de los asentamientos humanos y las rutas de escape de la población como los mostrados en la Figura 8. 

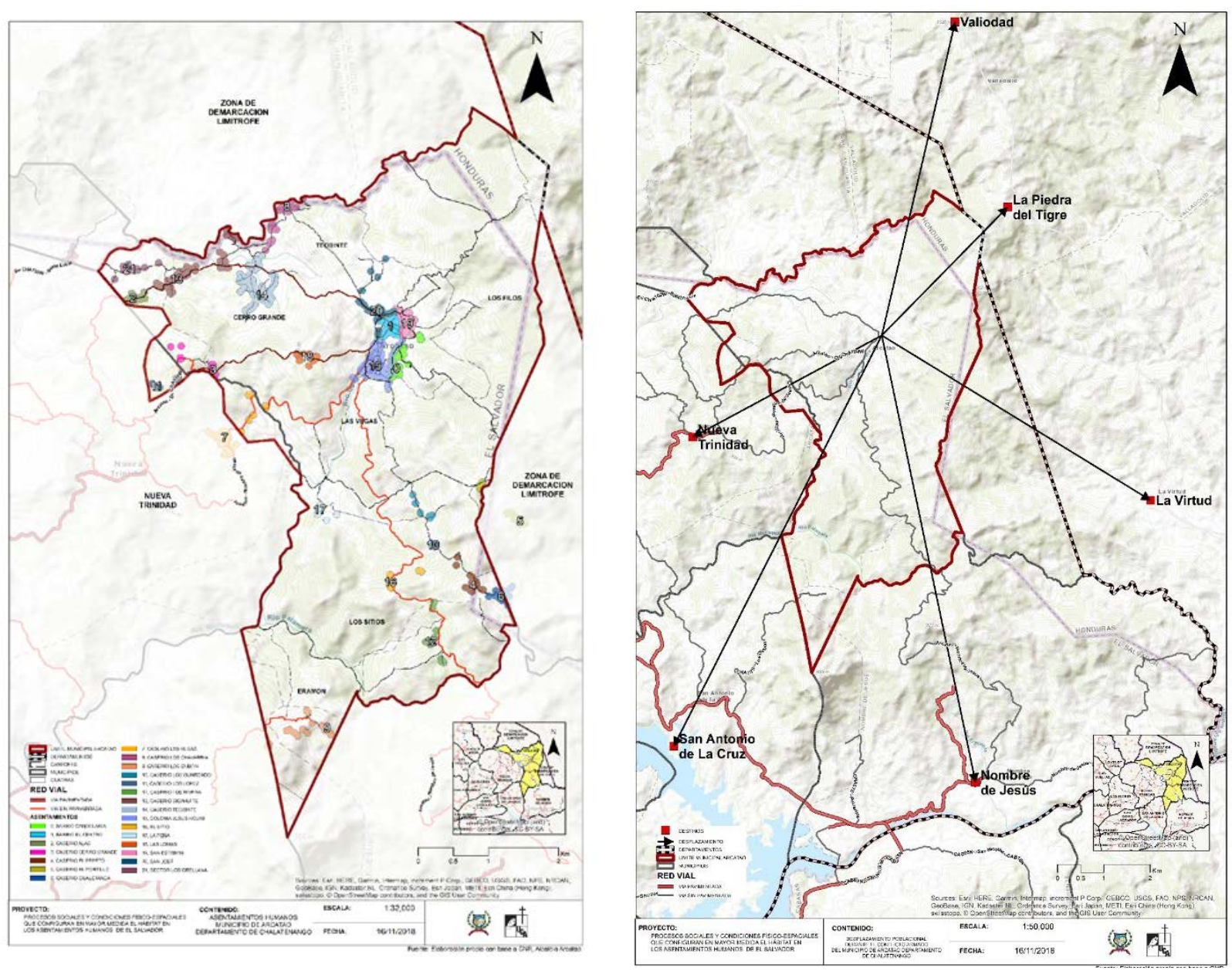

Figura 8: Inventario de asentamientos humanos y rutas de huida en Arcatao, Fuente Elaboración propia

\section{Conclusiones}

Es posible determinar al menos tres tipos de conclusiones, la primera es de tipo general y plantea al menos tres procesos sociales claves que posibilitan la transformación de las condiciones físico-espaciales: el primer proceso social clave es la organización comunitaria, del cual en Arcatao existen evidencias que está potenciada por que tiene a la base dos tipos de enfoques, el de tipo medioambiental y/o de reivindicación de derecho (Miranda Gassull, 2017), esto posibilita la aparición de un segundo proceso que denominamos autogestión (Carrillo, 2007, p. 24) y tiene como características la participación de diversos grados de la comunidad, capacitación de los beneficiarios y coordinadores así como la flexibilidad en la formulación de los proyectos. En tercer proceso clave es la construcción de infraestructuras del hábitat que no sean vivienda, sobre todo lo que tiene que ver con las redes pues como establece Dupuy (1998, p. 58) la integración de los núcleos poblados a las redes de la ciudad es un signo palpable de una verdadera conexión con la comunidad.

Un segundo bloque de conclusiones está centrado en los procesos sociales, destaca la influencia del contexto social en la producción del hábitat y aunque el primero dicta las pautas y tendencias a nivel nacional y local, la capacidad organizativa de la comunidad permite adaptar mejor dicho influjo, esta capacidad de adaptación es palpable cuando los grupos que surgen de la misma población reaccionan, se transforman, se especializan en diversos ámbitos y se reacomodan al contexto social imperante, tal situación es evidente para el caso de Arcatao, 
en la que los pobladores se adaptan y emergen en el tiempo con nuevas cualidades según sea el grado del fortalecimiento de sus capacidades dadas sus actividades de autogestión (véase Figura 9). En el ámbito de los procesos sociales los actores establecen relaciones entre si a partir de un tema aglutinante, articulándose y coordinándose desde un grupo gestor o un actor con destacadas cualidades respecto al conocimiento del territorio, recursos personales, económicos y liderazgo (Sen, 1982, 2017). Respecto al rol de los actores se puede establecer tres tipos, primero; pasivos a la espera del accionar de otros, segundo, reactivos a las convocatorias, se involucran y participan asumiendo tareas de coordinación más operativas que estratégicas y un tercer grupo relacionados con lo proactivo, estos asumen un rol más estratégico y aglutinante en la comunidad, finalmente se puede establecer que no todos los actores mantienen estos roles de manera uniforme, esto varía según los ámbitos e iniciativas en los que tienen necesidad, injerencia y/o especialización cada uno de los actores (véase tabla 5).

El tercer bloque de conclusiones es sobre las condiciones físico-espaciales del hábitat, el instrumento propone una estrategia de evaluación basado en lo "adecuado" o no de infraestructuras y procesos(Miranda Gassull, 2017, p. 224; ONU-HABITAT, 2012) y vuelve visible como las fuentes de financiamiento en el caso de El Salvador son escasas, inclusive durante el plan de reconstrucción post guerra, sin embargo, el enfoque permite establecer un rastro en la búsqueda de cooperantes internacionales y por lo tanto evidencia modelos de autogestión que permiten ir evolucionando los servicios (i.e. educación y salud). Es posible establecer la importante brecha que existe en cuanto al tema de conectividad principalmente por la ausencia de vías que vinculen a los asentamientos humanos, el instrumento revela lo clave de las redes en la mejora del hábitat y en el caso de Arcatao las condiciones de preexistencia de la topografía accidentada. A pesar de esto es posible determinar que el éxito en el desarrollo de la infraestructura es posible gracias a una organización comunitaria que autogestiona sus proyectos.

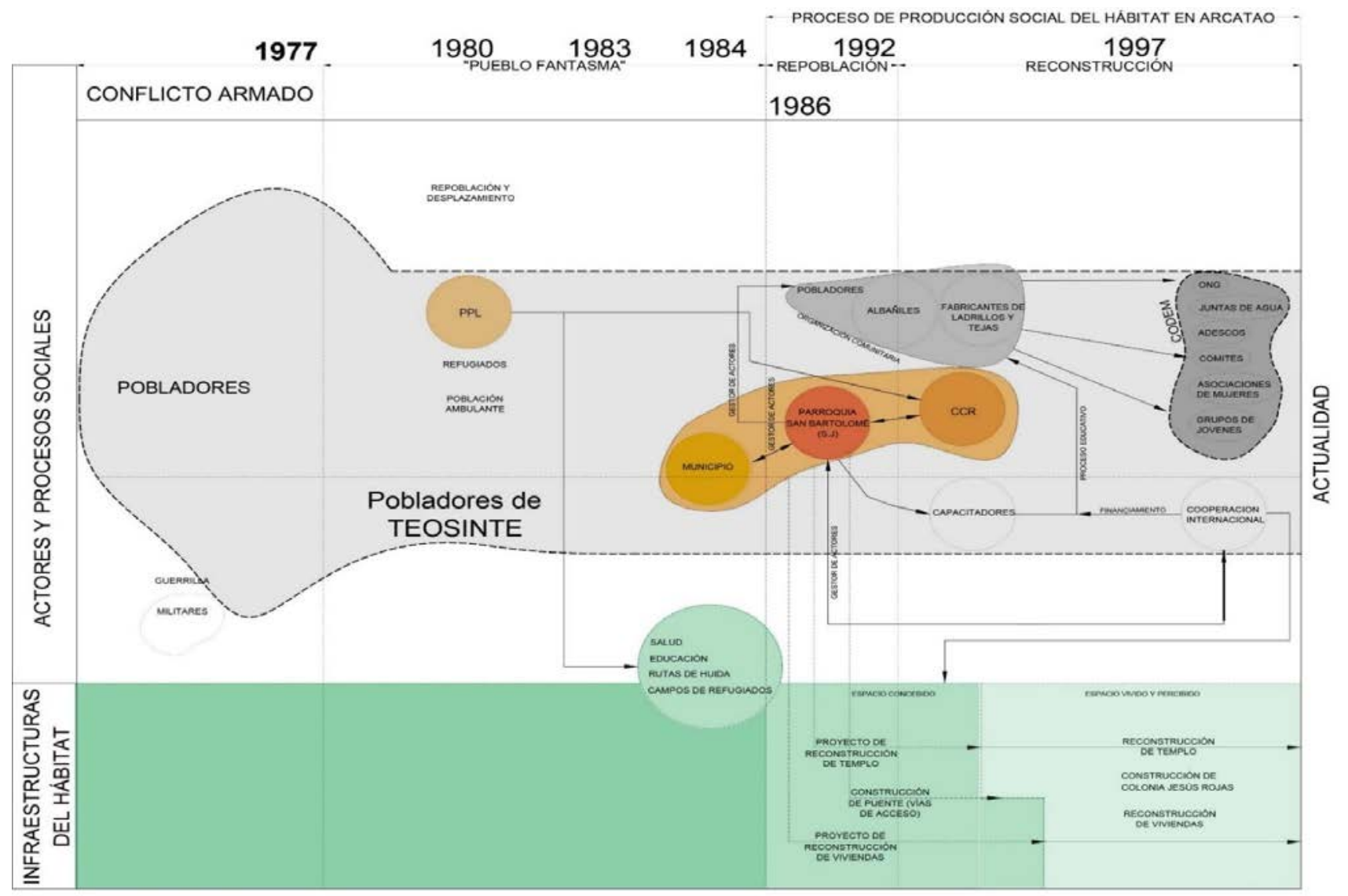

Figura 9: Desarrollo procesos sociales en la producción del hábitat. Fuente elaboración propia 


\section{BIBLIOGRAFÍA}

AGUIRRE, C. A. R. (2003). Contribución a la historia de la microhistoria italiana. Prohistoria: Manuel Suárez. ARCHER, M. S. (1995). Realist social theory: The morphogenetic approach. Cambridge university press. ASALE, R.-. (2014). Diccionario de la lengua española - Edición del Tricentenario.

BRAUN, V., \& CLARKE, V. (2006). Using thematic analysis in psychology. Qualitative research in psychology, 3(2), 77-101.

BRUGHA, R., \& VARVASOVSZKY, Z. (2000). Stakeholder analysis: a review. Health policy and planning, 15(3), 239-246.

CÁRDENAS, M., CORTÉS, F., ESCOBAR, A., NAHMAD, S., SCOTT, J., \& TERUEL, G. (2013). Manual para el Diseño y la Construcción de Indicadores. Instrumentos principales para el monitoreo de programas sociales de México. Consejo Nacional de Evaluación de la Política de Desarrollo Social, CONEVAL.

CARRILLO, D. (2007). Materiales básicos para el Proyecto Integral del Hábitat. Propuestas para El Salvador, C.A. (Fundación UPC).

DANERMARK, B., EKSTRÖM, M., JAKOBSEN, L., \& KARLSSON, J. C. (2016). Explicando la sociedad: el realismo crítico en las ciencias sociales. Antiguo Cuscatlán, La Libertad: UCA Editores.

DUPUY, G. (1998). El urbanismo de las redes: teorías y métodos.

ESPINOSA, N., \& VALDERRAMA, D. (2011). Pasos metodológicos para el análisis cuantitativo y cartográfico del conflicto armado en Colombia. Estudios Políticos, 39, 196-230.

HÁBITAT; NACIONES UNIDAS. (2016). La Nueva Agenda Urbana (p. 76).

HOLSTEIN, J. A., \& GUBRIUM, J. F. (1995). The active interview: En Qualitative research methods series 37. Thousand Oaks, CA: Sage Publication.

LEFEBVRE, H. (2013). La producción del espacio. Madrid: Captain Swing Madrid.

MIRANDA GASSULL, V. (2017). El hábitat popular. Algunos aportes teóricos de la realidad habitacional de sectores desposeídos. Territorios, (36), 217-238.

MOPTVDU. Política Nacional de Ordenamiento y Desarrollo Territorial. , (2007).

MOPTVDU. (2015, octubre 18). Política Nacional de Vivienda y Hábitat de El Salvador. Ministerio de Obras Públicas, Transporte y de Vivienda y Desarrollo Urbano.

ONU-HABITAT. (2012). Estado de las ciudades de América Latina y el Caribe. Rumbo a una nueva transición urbana -

ORTEGÓN, E., PACHECO, J., \& PRIETO, A. (2005). Metodología del marco lógico para la planificación, el seguimiento y la evaluación de proyectos y programas (CEPAL). En Manuales: Vol. 42 (CEPAL). Santiago de Chile: United Nations Publications.

ORTíZ, E., \& ZÁRATE, M. (2002). Vivitos y coleando: 40 años trabajando por el hábitat popular en América Latina. HIC-AL.

O'TOOLE, P., \& WERE, P. (2008). Observing places: using space and material culture in qualitative research. Qualitative research, 8(5), 616-634.

PEÑALVA, A., DE LOS RíOS, E., AGUILERA, S., \& ERASO, L. (2014). Manual de Participación en Políticas de Movilidad y Desarrollo Urbano (p. 143) [Manual de prácticas]. 
RODRÍGUEZ, M. C., DI VIRGILIO, M., PROCUPEZ, V., VIO, M., GAUTHIER, F. LVARO O., MENDOZA, M., \& MORALES, B. (2007). Producción social del hábitat y políticas en el rea Metropolitana de Buenos Aires: historia con desencuentros. Documentos de Trabajo (Universidad de Buenos Aires. Facultad de Ciencias Sociales), (49), 1.

ROUSSOS, A. (2011). Preparación de una revisión bibliográfica para su publicación Cuando un solo artículo nos habla de muchos trabajos Reportes de investigación, Junio 2011, 1: 1-7. Reportes de investigación, Universidad de Belgrano, CONICET, FUNICS.

SCOTT, J. (2017). Social Network Analysis. SAGE.

SEN, A. (1982). Rights and Agency. Philosophy \& Public Affairs, 11(1), 3-39.

SEN, A. (2017, mayo 15). Well-Being, Agency and Freedom the Dewey Lectures 1984 *. https://doi.org/10.4324/9781315251240-1

SOTO BAQUERO, F., BEDUSCHI FILHO, L. C., DIEZ DE SOLLANO, R., VALENZUELA CHADWICK, C., \& PALMA, A. (2007). Desarrollo territorial rural: análisis de experiencias en Brasil, Chile y México. Inter-American Development Bank.

ZÚÑIGA, A. (2009). Indicadores para la evaluación de la calidad ambiental del hábitat urbano. Nexo, 22(01), 2331. 